\title{
High transmittance of X-rays in the utricular otolith of upside-down swimming catfish, Synodontis nigriventris
}

\author{
Ken Ohnishi ${ }^{1}$, Toshihiro Yamamoto ${ }^{2}$, Yoichi Ogawa ${ }^{3}$, Akihisa Takahashi ${ }^{1}$, Masayuki Yamashita ${ }^{3}$ and Takeo Ohnishi $^{1}$ \\ Departments of ${ }^{1}$ Biology, ${ }^{2}$ Otorhinolaryngology and ${ }^{3}$ Physiology Nara Medical University, 840 Shijo-cho, Kashihara, \\ Nara 634-8521, Japan
}

\begin{abstract}
The upside-down swimming catfish (Synodontis nigriventris) has unique behavior, i.e., it frequently shows a stable upside-down posture during swimming and resting. To examine whether the unique postural control in S. nigriventris results from the characteristics of the vestibular organ, we observed the morphological aspects of the otolith and the orientation of sensory hair cells in the utricle. Soft X-ray densitometry analysis showed that the transmittance of soft X-rays in the otolith of S. nigriventris was higher than that in a closely related species (Synodontis multipunctatus) belonging to Synodontis family, goldfish (Carassius auratus) or miniature catfish (Corydoras paleatus) which shows upside-up swimming. The higher transmittance of soft $\mathrm{X}$-rays suggests that the density of the otolith in $S$. nigriventris is lower than that in $S$. multipunctatus, $C$. auratus or $C$. paleatus. It is possible that the low density of the otolith may have a relation to the control of the unique upside-down posture of $S$. nigriventris. The hair cells in $S$. nigriventris were present at the ventral to ventro-lateral site of the utricular epithelium, forming a single hair cell layer as in the other 3 species of fish. The orientation of the sensory hair cells does not appear to cause the unique postural control.
\end{abstract}

Key words: upside-down swimming catfish, postural control, utricular otolith, X-ray densitometry, microscopic analysis

\section{Introduction}

As their name implies, upside-down swimming catfish (Synodontis nigriventris) frequently swim with the dorsal surface down (Meyer et al., 1976; Nagaishi et al., 1989; Chapman et al., 1994; Ohnishi et al., 1996a, b). We assume that the posture of $S$. nigriventris is controlled by unique postural control system different from that of other fishes. The vestibular organ plays an important role in postural control of fishes, especially their sense of balance (Lowenstein, 1971, 1974; Platt, 1983). We have previously reported the effects of removal of the vestibular organ on postural control in S. nigriventris (Ohnishi et al., 1999). The operated $S$. nigriventris could not keep the stable swimming posture under rotating water flow generated with a clinostat. The utricle in fishes is a balance detector, while the saccule and the lagena are sound detectors from behavioral studies (von Frisch, 1938; Schöen and von Holst, 1950; von Holst, 1950). The nature of the saccule and the lagena as sound detector has been confirmed by neurophysiological studies (Furukawa and Ishii, 1967; Fay, 1978, 1984) and a neuroanatomical study using goldfish (McCormick and Braford, 1994). Thus, the characteristics of the utricle were examined in the present study. No histological study on the vestibular organ in S. nigriventris has been performed yet except for a report sketching the

Original paper

Received December 17, 2001

Accepted March 25, 2002

Address for correpondence: Takeo Ohnishi Department of Biology, Nara Medical University 840 Shijo-cho, Kashihara, Nara 634-8521, Japan

E-mail: tohnishi@naramed-u.ac.jp macro-shape of the vestibular organ (Meyer et al. 1976). Here, we examined the characteristics of the utricle of $S$. nigriventris and 3 species of fish showing upside-up swimming.

\section{Materials and Methods}

Animals

Upside-down swimming catfish (Synodontis nigriventris), a closely related catfish (Synodontis multipunctatus), goldfish (Carassius auratus) and miniature catfish (Corydoras paleatus) of 3-4 cm in body length, which were obtained from a local dealer (Kashihara, Nara, Japan), were used in the present study. They were maintained in aquariums at water temperature of $23-25^{\circ} \mathrm{C}$.

Removal of otolith

Fishes were anesthetized by putting them in beakers containing $0.1 \%$ tricaine methanesulfonate (MS222, Sigma, St. Louis, MO, USA) and then were fixed on cork boards under a binocular microscope. A part of the skull was opened with a dental drill (LMM-7, Morita, Tokyo, Japan) and the otolith in the right and left utricles was picked out with tweezers.

\section{Measurement of otolith and body weights}

The removed otoliths were dehydrated with methanol, dried overnight, and weighed. The mean weight obtained from right and left otoliths was used for data analysis. The weight of the body was measured under the anesthetized condition prior to the removal of the otoliths.

Soft $X$-ray analysis

The dried right and left otoliths were irradiated by use of a soft X-ray apparatus (Softex E-SM, Koizumi, Tokyo, Japan) at $60 \mathrm{~V}$ and $2.5 \mathrm{~mA}$ for various duration $(1,2,3,4$ 
or $6 \mathrm{sec}$ ). X-ray films (IX-FR, FUJIFILM, Tokyo, Japan), on which otolith image was printed, were used for the measurement of otolith density. Otolith density was measured with the public domain NIH Image program. The relative density of otolith was obtained as a ratio of otolith density of $S$. nigriventris, $S$. multipunctatus or $C$. paleatus to that of $C$. auratus at each dose of irradiation.

\section{Scanning electron microscopic observation}

The otoliths isolated from the utricles of the 4 species of fish were rinsed with phosphate buffer and provided for the observation by use of a scanning electron microscope (S-3200N, Hitachi, Tokyo, Japan).

\section{Preparation of frontal sections}

Fishes anesthetized with MS222 were decapitated. After fixation with Bouin solution for 7 days and decalcifiction with 10\% EDTA for 3-5 days, the head parts were embedded in paraffin and sectioned at 5-10 $\mu \mathrm{m}$ in thickness with cryostat. After deparaffinization with xylene for 2 days, the sections were stained with $0.05 \%$ luxol fast blue for $6 \mathrm{~h}$ and with $0.1 \%$ cresyl violet for $30 \mathrm{~min}$ and mounted with resin.

\section{Results}

Measurement of otolith and body weight

The weight of a pair of otolith and body was measured in 5 Synodontis nigriventris, 5 Synodontis multipunctatus, 4 Corydoras paleatus and 20 Carassius auratus. A relationship between the otolith and body weight is shown in Fig. 1. Individual body weight of $S$. nigriventris was

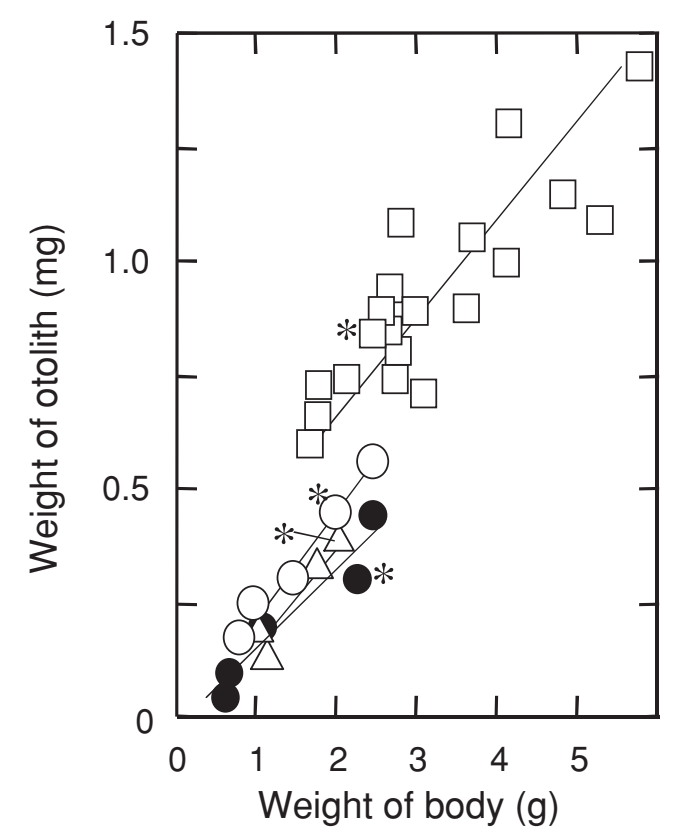

Fig. 1 Relationship between otolith in the utricle and body weight. Each symbol indicates mean value of left and right otolith weight. Filled circles, Synodontis nigriventris. Open circles, Synodontis multipunctatus. Open triangles, Corydoras paleatus. Open squares, Carassius auratus. Asterisked samples were used for X-ray densitometry in Fig. 2 and the scanning microscopy observation in Fig. 3.
0.6 to $2.3 \mathrm{~g}$, and the corresponding otolith weight (mean value of right and left otoliths) was 0.1 to $0.4 \mathrm{mg}$. Similarly, individual body weight of $S$. multipunctatus and C. paleatus was 0.8 to $2.3 \mathrm{~g}$ and 1.0 to $2.0 \mathrm{~g}$ and the corresponding otolith weight was 0.2 to $0.6 \mathrm{mg}$ and 0.2 to $0.4 \mathrm{mg}$, respectively. The relationship between the otolith and body weight was very similar between $S$. nigriventris, $S$. multipunctatus and C. paleatus. Contrarily, the otolith weight of $C$. auratus was relatively large compared with that of the other 3 species in spite of similar body weight to that of 3 species. Individual body weight of $C$. auratus was 2.2 to $5.8 \mathrm{~g}$ and the corresponding otolith weight was 0.6 to $1.4 \mathrm{mg}$.

Soft $X$-ray analysis of otoliths

The otoliths removed from the 4 species of fish (asterisked samples in Fig. 1) were provided for soft X-ray irradiation. The X-ray films were used for the densitometry analysis. The relative density of otoliths in S. nigriventris (0.1-0.5, the density of otolith in $C$. auratus $=1$, mean value of right and left otoliths) was smaller than that (0.6-0.8) in $S$. multipunctatus or C. paleatus and the difference in the density among them became larger with increase in the duration of soft X-ray irradiation (Fig. 2).

\section{Scanning electron microscopic observation}

Morphology of the otoliths of the 4 species of fish (asterisked samples in Fig. 1) was observed by use of the scanning electron microscopy. The shape of the otolith was very different among the 4 species, as shown in Fig. 3, but it was similar among individuals in each species. The otolith size of $C$. auratus was about 2-fold (about $1.0 \times 2.0 \mathrm{~mm}$ )

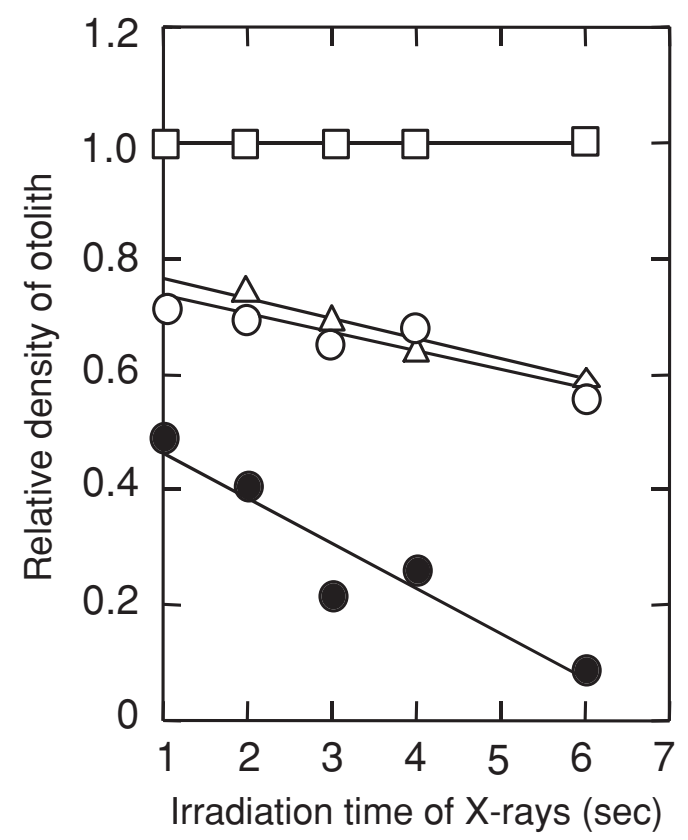

Fig. 2 Transmittance of soft X-rays in utricular otoliths. The asterisked samples in Fig. 1 were irradiated with soft X-rays at $60 \mathrm{~V}$ and $2.5 \mathrm{~mA}$. The density of otolith image on X-ray film was measured by use of a densitometer (each symbol, mean value of right and left otolith). Filled circles, Synodontis nigriventris. Open circles,Synodontis multipunctatus. Open triangles, Corydoras paleatus. Open squares, Carassius auratus. 
larger than that of $S$. nigriventris (about $0.6 \times 1.0 \mathrm{~mm}$ ), $S$. multipunctatus (about $0.6 \times 1.0 \mathrm{~mm}$ ) or $C$. paleatus (about $0.7 \times 1.2 \mathrm{~mm})$ despite their similar body weight (2.0-2.2 $\mathrm{g})$. The thickness of the otolith in its central region was about 590, 400, 410 and $750 \mu \mathrm{m}$ in $S$. nigriventris, $S$. multipunctatus, C. paleatus and $C$. auratus, respectively. The surface of the ventral side of the otolith (Fig. 3b, d, f and $h$ ) in all 4 species was rough compared that of the dorsal side (Fig. 3a, c, e and g), especially in S. nigriventris.

Light microscopic observation of the utricle

The frontal sections of the utricles in $S$. nigriventris, $S$. multipunctatus, $C$. auratus and $C$. paleatus were stained with luxol fast blue and cresyl violet and observed by use of a light microscope. The shape of the utricle in $S$. nigriventris was elliptic to the frontal plane (Fig. 4a). The otolith in S. nigriventris was also elliptic and located on the ventral side of the utricle. The sensory hair cells were cylinder- or gourd-shape at the ventrolateral site of the utricular epithelium and large in size (Fig. 4c) and pear- or eggplant-shape at the ventral site and small (Fig. 4d), and contacted the bottom of the otolith with their sensory hairs. These morphological characteristics of the utricle and the hair cells were similarly observed in S. multipunctatus (Fig. $4 \mathrm{~b}$, e and f) and the other 2 species.

\section{Discussion}

Several reports (Meyer et al., 1976; Nagaishi et al., 1989; Chapman et al., 1994; Ohnishi et al., 1996a, b) have previously shown that Synodontis nigriventris has a unique habit that it frequently maintains its posture with stable upside-down posture. However, it remains unknown what elements control this unique behavior, because systematic analyses of the neural control mechanism have not yet been performed. Vestibular organs of fishes are well known to play an important role in their postural control. The slippage of otoliths involved in the vestibular organs triggers balance sensation. Thus, we examined in the present study whether the unique swimming behavior of $S$. nigriventris results from the specific nature of its otolith. First, the weight of utricular otoliths isolated from 4 species of fish $(S$. nigriventris, Synodontis multipunctatus, Carassius auratus and Corydoras paleatus) was measured. The ratio of otolith weight to body weight was smaller in S. nigriventris than in $C$. auratus but was almost equal to that in $S$. multipunctatus or $C$. paleatus. It seems that the weight of otolith is not an important factor for postural control of the upside-down posture. The difference in the ratio among them may come from their habit. C. auratus usually swims or rests in a free area of water, actually maintaining its body balance by manipulating its fins, while $S$. multipunctatus and $C$. paleatus usually rest at the bottom of water touching their ventral side onto the bottom, and showing no manipulative movements of their fins. $S$. nigriventris also usually tends to rest with touching its ventral side on the surface of some objects. The weight of the otolith may be correlated with their postural control pattern.
Secondly, to estimate the density of structure of otoliths, soft X-ray analysis was performed. The otoliths of 4 species of fish (asterisked samples in Fig. 1) were irradiated with soft X-rays and their images were printed on X-ray films. Despite the similarity of otolith size in S. nigriventris and $S$. multipunctatus or $C$. paleatus, the transmittance of soft $\mathrm{X}$-rays in otoliths of $S$. nigriventris was higher than that of $S$. multipunctatus or $C$. paleatus. Since the thickness of the otolith was similar among $S$. nigriventris and $S$. multipunctatus or $C$. paleatus with a slight larger its thickness in S. nigriventris, it is suggested that the higher $\mathrm{X}$-ray transmittance in $S$. nigriventris does not result from the thin thickness of the otolith. The higher X-ray transmittance suggests that the density of the otoliths of $S$. nigriventris may be lower than in that of $S$. multipunctatus or $C$. paleatus. The specific gravity of the otolith seems to be small judging from the low density of otolith. The low specific gravity may be closely related to the unique postural control of $S$. nigriventris. However, it is unknown at present whether the low specific gravity is a cause or effect of the upside-down posture control. There remain other possible mechanisms relating to the postural control, such as neural integration in the central nervous system and central programmed motor patterns after receiving sensory inputs.

During upside-down swimming, the direction of gravity relative to the upside-down catfish is reversed. Although a previous study showed that the macro-structure of the vestibular organ of $S$. nigriventris is similar to that of other fishes (Meyer et al., 1976), we presumed that the microstructure of the utricle in S. nigriventris would be different from that of other fishes. That is, we hypothesized that the sensory hair cells of the utricle might be present on both the dorsal and ventral sides, and that the otolith could therefore bilaterally contact the hair cells. To test this hypothesis, thirdly, we observed the orientation of sensory

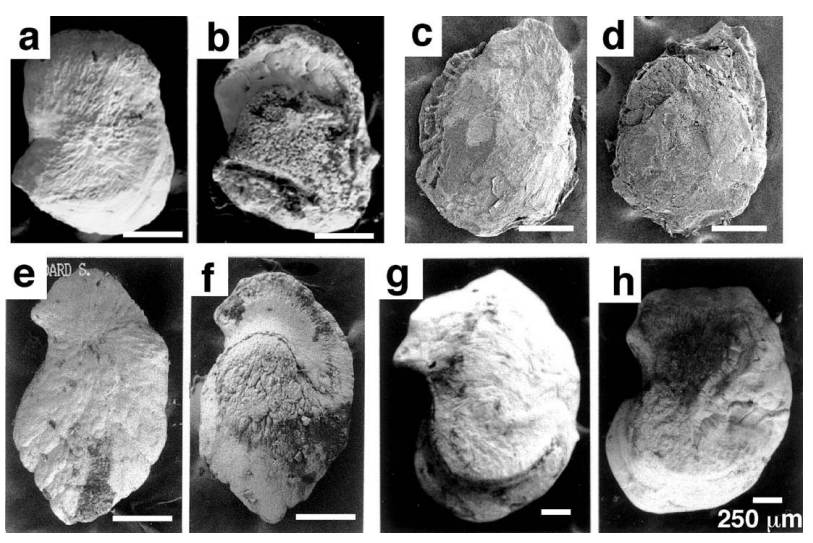

Fig. 3 Scanning microscopy observation of otoliths in the utricle. a and b, Synodontis nigriventris; $\mathbf{c}$ and $\mathbf{d}$, Synodontis multipunctatus; $\mathbf{e}$ and $\mathbf{f}$, Corydoras paleatus; $\mathbf{g}$ and $\mathbf{h}$, Carassius auratus. a, c, e and $\mathbf{g}$, dorsal view of right otolith; $\mathbf{b}, \mathbf{d}, \mathbf{f}$ and $\mathbf{h}$, ventral view of left otolith. The upper and lower sides of the otoliths correspond to the inner and outer sides of the utricle, respectively. The asterisked samples in Fig. 1 were used for the scanning microscopy observation (mean values in weight of $\mathbf{a}$ and $\mathbf{b}$, $\mathbf{c}$ and $\mathbf{d}, \mathbf{e}$ and $\mathbf{f}, \mathbf{g}$ and $\mathbf{h}=0.30,0.45,0.35,0.85 \mathrm{mg}$ ). 

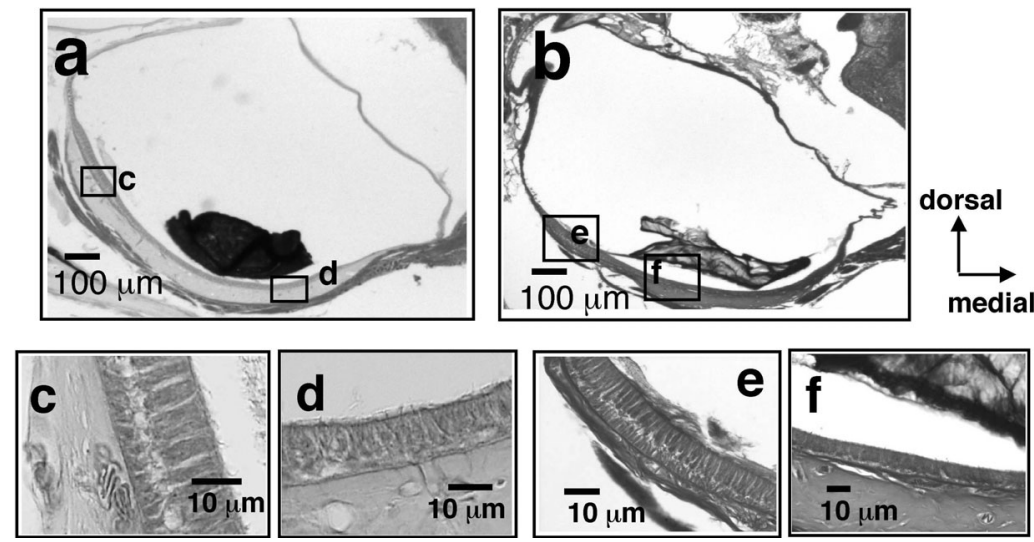

Fig. 4 Frontal sections of the utricle in 2 species of Synodontis family. a, Synodontis nigriventris; $\mathbf{b}$, Synodontis multipunctatus; $\mathbf{c}$ and $\mathbf{e}$, cylinder- or gourd-shape large hair cells at the ventrolateral site; $\mathbf{d}$ and $\mathbf{f}$, pear- or eggplant-shape small hair cells at the ventral site.

hairs of the utricle. Contrary to our presumption, the hair cells were present on only the ventral to ventrolateral side of the utricular epithelium, forming a single cell layer. Some sensory hair cells contacted the otolith with their hairs. The ventral side of the otolith was rather rough compared with the dorsal side, from the scanning electron microscopic observation (Fig. 3). This roughness well correlated with driving the movement of sensory hairs due to effectively hooking the sensory hairs onto the surface of the otolith. From these observations, the structural characteristics of the utricle do not cause upside-down swimming. We did not perform light microscopic observation of the sensory hair cells of the saccule and the lagena, with which otoliths are involved. Thus, there remains the possibility that these organs may contribute to the postural control in S. nigriventris. However, since the saccule and the lagena in other fishes contribute to auditory sense (von Frisch, 1938; Schöen and von Holst, 1950; von Holst, 1950; Furukawa and Ishii, 1967; Fay, 1978, 1984; McCormick and Braford, 1994), this possibility seems to be weak. To ascertain this possibility, we have been studying on the histological characteristics of saccule and the lagena of $S$. nigriventris in detailed.

In conclusion, we suggest that the density of utricular otolith in S. nigriventris is lower than that in other fishes showing upside-up swimming posture. This feature of the otolith may have a relation to the unique postural control in S. nigriventris. However, further investigations are required for the understanding of the central nervous system after receiving sensory information from the vestibular organ.

\section{Acknowledgments}

This study was supported by "Space Utilization Frontiers Joint Research Projects" promoted by NASDA and "Fund for basic experiments oriented to space station utilization" by the Institute of Space and Astronautical Science.

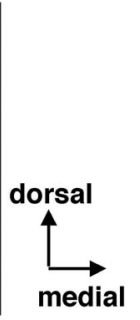

\section{References}

Chapman, L.J., Kaufman, L. and Chapman, C.A. (1994) Why swim upside down?: A comparative study of two mochokid catfishes. Copeia, 1994, 130-135.

Fay, R.R. (1978) Coding of information in single auditory-nerve fibers of the goldfish. J. Acoust. Soc. Am., 63, 136-146.

Fay, R.R. (1984) The goldfish ear codes the axis of acoustic particle motion in three dimensions. Science, 225, 951-954.

Furukawa, T. and Ishii, Y. (1967) Neurophysiological studies on hearing in goldfish. J. Neurophysiol., 30, 1377-1403.

Lowenstein, O. (1971) The labyrinth. In Fish Physiology. Sensory Organs, vol. 5 (ed. W.S. Hoar \& D.J. Randall) pp. 207-240. New York, Academic Press.

Lowenstein, O. (1974) Comparative morphology and physiology. In Vestibular System: Basic Mechanisms; Handbook of Sensory Physiology, vol. 6 (ed. H.H. Kornhuber) pp. 75-120. New York, Springer-Verlag.

McCormick, C.A. and Braford, M.R. Jr. (1994) Organization of inner ear endorgan projections in the goldfish, Carassius auratus. Brain Behav. Evol., 43, 189-205.

Meyer, D.L., Platt, C. and Distel, H.-J. (1976) Postural control mechanisms in the upside-down catfish. J. Comp. Physiol., 110, 323-331.

Nagaishi, H., Nishi, H., Fujii, R. and Oshima, N. (1989) Correlation between body color and behavior in the upsidedown catfish, Synodontis nigriventris. Comp. Biochem. Physiol., 92A, 323-326.

Ohnishi, K., Takahashi, A., Tanaka, H. and Ohnishi, T. (1996a) Relationship between frequency of upside-down posture and space size around upside-down catfish, Synodontis nigriventris. Biol. Sci. Space, 10, 247-251.

Ohnishi, K., Takahashi, A., Koyama, M. and Ohnishi, T. (1996b) Postural control under clinorotation in upside-down catfish, Synodontis nigriventris. Biol. Sci. Space, 10, 252-258.

Ohnishi, K., Yamamoto, T., Takahashi, A., Tanaka, T., Koyama, M. and Ohnishi, T. (1999) The effect of labyrinthectomy on postural control of upside-down swimming catfish, Synodontis nigriventris, under pseudo-microgravity. Physiol. Behav., 67, 89-93.

Platt, C. (1983) The peripheral vestibular system of fishes. In Fish Neurobiology, Brainstem and Sensory Organs, vol. 1 (ed. R.G. Northcutt \& R.E. Davis) pp.89-123. Ann. Arbor, University of Michigan Press.

Schöen, L. and von Holst E. (1950) Das Zusammenspiel von Lagena und Utriculus bei der Lageorientierung der Knochenfishe. Z. vergl. Physiol., 32, 552-571.

von Frisch, K. (1938) Über die Bedeutung des Sacculus und der Lagena für den Gehörsinn der Fische. Z. vergl. Physiol., 25, 703-747.

von Holst, E. (1950) Die Arbeitsweise des Statolithenapparates bei Fischen. Z. vergl. Physiol., 32, 60-120. 